\title{
RANDOM DATA CAUCHY THEORY FOR SUPERCRITICAL WAVE EQUATIONS I: LOCAL THEORY
}

\author{
by
}

Nicolas Burq \& Nikolay Tzvetkov

\begin{abstract}
We study the local existence of strong solutions for the cubic nonlinear wave equation with data in $H^{s}(M), s<1 / 2$, where $M$ is a three dimensional compact riemannian manifold. This problem is supercritical and can be shown to be strongly ill-posed (in the Hadamard sense). However, after a suitable randomization, we are able to construct local strong solution for a large set of initial data in $H^{s}(M)$, where $s \geq 1 / 4$ in the case of a boundary less manifold and $s \geq 8 / 21$ in the case of a manifold with boundary.
\end{abstract}

\section{Introduction}

In the study of the local well-posedness of a nonlinear evolutionary PDE, one often encounters the presence of a critical threshold for the well-posedness theory. A typical situation is to have a method showing well-posedness in Sobolev spaces $H^{s}$ where s is greater than a critical index $s_{c r}$. This index is often related to a scale invariance (leading to solutions concentrating at a point of the space-time) of the considered equation. In some cases (but not all), a good local well-posedness theory is valid all the way down to the scaling regularity. On the other hand, at least in the context of nonlinear dispersive equations, no reasonable local well-posedness theory is known for any supercritical equation, i.e. for data having less regularity than the scaling one. In fact, recently, several methods to show ill-posedness, or high frequency instability, for $s<s_{c r}$ emerged (see the works by Burq, Gérard and Tzvetkov [6, 5, Lebeau [12 and Christ Colliander and Tao [10). The goal of this paper is to give a class of equations for which, using probabilistic arguments, one can still obtain a suitable well-posedness theory below the critical threshold. Our model will be the cubic nonlinear wave equation posed on a compact manifold.

2000 Mathematics Subject Classification. - 35Q55, 35BXX, 37K05, 37L50, 81Q20 .

Key words and phrases. — nonlinear wave equation, eigenfunctions, dispersive equations. 
Let $(M, g)$ be a three dimensional compact smooth riemannian manifold (without boundary) and let $\boldsymbol{\Delta}$ be the Laplace-Beltrami operator associated to the smooth metric $g$. For $s \in \mathbb{R}$, we denote by $H^{s}(M)$ the classical Sobolev space equipped with the norm $\|u\|_{H^{s}(M)}=\left\|(1-\boldsymbol{\Delta})^{s / 2} u\right\|_{L^{2}(M)}$. Consider the following cubic wave equation

$$
\left(\partial_{t}^{2}-\boldsymbol{\Delta}\right) u+u^{3}=0,\left.\quad\left(u, \partial_{t} u\right)\right|_{t=0}=\left(f_{1}, f_{2}\right)
$$

with real valued initial data $\left(f_{1}, f_{2}\right) \equiv f \in H^{s}(M) \times H^{s-1}(M) \equiv \mathcal{H}^{s}(M)$.

Using Strichartz estimates for the free evolution (see Section 2) one can show that for $s>1 / 2$ the Cauchy problem (1.1) is locally well-posed for data in $\mathcal{H}^{s}(M)$. This means that for every $f \in \mathcal{H}^{s}(M)$ there exists $T>0$ and a unique solution $u$ of (1.1), in a suitable class, such that $\left(u, u_{t}\right) \in C\left([0, T] ; \mathcal{H}^{s}(M)\right)$, i.e. the solution $u$ represents a continuous curve in $H^{s}(M)$ (we call such a solution strong solution since the classical construction of weak solutions does not yield the continuity in time). Moreover, we can show that the time existence $T$ may be chosen the same for all $f$ belonging to a fixed bounded set $B$ of $\mathcal{H}^{s}(M)$ and the map $f \mapsto\left(u, u_{t}\right)$ is continuous (and even Lipschitz continuous) from $B$ to $C\left([0, T] ; \mathcal{H}^{s}(M)\right)$.

For $s=1 / 2$ one can still construct local strong solution for $f \in \mathcal{H}^{1 / 2}(M)$ but the dependence of $T$ on $f$ is more complicated and the Sobolev space $\mathcal{H}^{1 / 2}(M)$ is called critical space for (1.1).

For $s<1 / 2$, the argument to construct local solutions by Strichartz estimates breaks down. Moreover one may show (see [10, [12] for the case of constant coefficient metrics or the appendix of this paper for the case of non constant coefficient metrics ) that if the initial data belong to $\mathcal{H}^{s}(M), s<1 / 2$, the Cauchy problem (1.1) is ill-posed in a strong sense: there exists initial data $\left(f_{1}, f_{2}\right) \in \mathcal{H}^{s}(M)$ such that any reasonable solution of (1.1), i.e. satisfying the finite speed of propagation ceases instantaneously to be in $\mathcal{H}^{s}$ for positive times (by finite speed of propagation, we mean the fact that the value of the solution at $\left(x_{0}, t_{0}\right)$ depends only on the values of the initial data on the set of points located at distance smaller that $\left.\left|t_{0}\right|:\left\{x: d_{g}\left(x, x_{0}\right) \leq\left|t_{0}\right|\right\}\right)$. However, the functions for which one can prove such a pathological behavior are highly non generic and a natural question is whether despite this result one can still prove that the problem (1.1) possesses local strong solutions for a "large class of functions" in $\mathcal{H}^{s}(M), s<1 / 2$. Our purpose in this paper is precisely to give a positive answer to this question. Let us observe that the possibility of such a phenomenon in the context of the nonlinear Schrödinger equation (NLS) is studied in the last section of [17. However, the situation in the context of NLS is much more involved and it would be interesting to decide whether the main result of this paper has an appropriate extension in the context of NLS (or other nonlinear PDE's).

Let us first precise what we mean by "a large class of initial data in $\mathcal{H}^{s}(M)$ ". Let $e_{n} \in C^{\infty}(M), n=1,2, \ldots$ be an orthonormal basis of $L^{2}(M)$ constructed from real eigenfunctions of the operator $-\boldsymbol{\Delta}$ associated to eigenvalues $\lambda_{n}^{2}$. Let $\left(\left(h_{n}(\omega), l_{n}(\omega)\right)_{n=1}^{\infty}\right.$ 
be a sequence of independent, 0 mean value, real random variables on a probability space $(\Omega, \mathcal{A}, p)$ such that

$$
\exists C>0: \forall n \geq 1, \int_{\Omega}\left(\left|h_{n}(\omega)\right|^{4}+\left|l_{n}(\omega)\right|^{4}\right) d p(\omega)<C .
$$

For $f=\left(f_{1}, f_{2}\right) \in \mathcal{H}^{s}(M)$ given by

$$
f_{1}(x)=\sum_{n=1}^{\infty} \alpha_{n} e_{n}(x), \quad f_{2}(x)=\sum_{n=1}^{\infty} \beta_{n} e_{n}(x), \quad \alpha_{n}, \beta_{n} \in \mathbb{R},
$$

we consider the map

$$
\omega \longmapsto f^{\omega}=\left(f_{1}^{\omega}, f_{2}^{\omega}\right)
$$

from $(\Omega, \mathcal{A})$ to $\mathcal{H}^{s}(M)$ equipped with the Borel sigma algebra, defined by

$$
f_{1}^{\omega}(x)=\sum_{n=1}^{\infty} h_{n}(\omega) \alpha_{n} e_{n}(x), \quad f_{2}^{\omega}(x)=\sum_{n=1}^{\infty} l_{n}(\omega) \beta_{n} e_{n}(x) .
$$

Using (1.2) one can check that the map $\omega \mapsto f^{\omega}$ is measurable and $f^{\omega} \in L^{2}\left(\Omega ; \mathcal{H}^{s}(M)\right)$. Thus it defines a $\mathcal{H}^{s}(M)$ valued random variable. A simple computation (see Appendix 2) shows that if $h_{n}, l_{n}$ are identically distributed and different from zero, or more generally if there exists $c>0$ such that the distributions $h_{n}, l_{n}$ satisfy

$$
\limsup _{n \rightarrow+\infty} p\left(\left\{\left|h_{n}\right|+\left|l_{n}\right| \leq c\right\}\right)<1,
$$

then, if $f$ does not belong to $\mathcal{H}^{s+\varepsilon}(M)$, for almost all $\omega, f^{\omega}$ does not belongs to $\mathcal{H}^{s+\varepsilon}(M)$. Thus the randomization $\omega \mapsto f^{\omega}$ does not give a regularization in the scale of the Sobolev spaces (but we shall crucially exploit $L^{p}$ regularizations of this randomization). Our main result reads as follows.

Theorem 1. - Assume that $\partial M=\emptyset$. Let us fix $s \geq \frac{1}{4}$ and $f=\left(f_{1}, f_{2}\right) \in \mathcal{H}^{s}(M)$. Let $f^{\omega} \in L^{2}\left(\Omega ; \mathcal{H}^{s}(M)\right)$ be defined by the randomization (1.3), (1.4). Then there exists $\sigma \geq \frac{1}{2}$ such that for almost all $\omega \in \Omega$ there exist $T_{\omega}>0$ and a unique solution to (1.1) with initial data $f^{\omega}$ in a space continuously embedded in

$$
X_{\omega}=\left(\cos (t \sqrt{-\boldsymbol{\Delta}}) f_{1}^{\omega}+\frac{\sin (t \sqrt{-\boldsymbol{\Delta}}) f_{2}^{\omega}}{\sqrt{-\boldsymbol{\Delta}}}\right)+C\left(\left[-T_{\omega}, T_{\omega}\right] ; H^{\sigma}(M)\right) .
$$

More precisely, there exist $C>0, \delta \geq 0$ ( $\delta>0$ if $s>1 / 4$ ) and for every $0<T \leq 1$, an event $\Omega_{T}$ such that

$$
p\left(\Omega_{T}\right) \geq 1-C T^{1+\delta}
$$

and such that for every $\omega \in \Omega_{T}$ there exists a unique solution $u$ of (1.1) with data $f^{\omega}$ in a space continuously embedded in $C\left([-T, T] ; H^{s}(M)\right)$. 
Moreover, if $s>1 / 4$ and $h_{n}, l_{n}$ are standard real Gaussian or Bernoulli variables (1.5) can be improved to

$$
p\left(\Omega_{T}\right) \geq 1-C e^{-c / T^{\delta}}, \quad c, \delta>0 .
$$

Let us make several remarks.

Remark 1.1. - The result in Theorem 1 shows that in some sense the problem (1.1) is well-posed for almost all initial data in $\mathcal{H}^{\frac{1}{4}}(M)$, exhibiting a gain of $1 / 4$ derivatives with respect to the critical index $1 / 2$.

Remark 1.2. - For any $f \in \mathcal{H}^{s}(M), 1 / 4 \leq s$, the map

$$
\omega \in \Omega \mapsto f^{\omega} \in \mathcal{H}^{s}(M)
$$

endows naturally $\mathcal{H}^{s}(M)$ with a probability measure $\mu_{f}$. It is straightforward to check that the solutions given by Theorem 1 satisfy the finite speed of propagation. As a consequence, Theorem 1 implies that the set of initial data exhibiting the same kind of pathological behavior as the ones we constructed in the appendix have measure 0 for any measure $\mu_{f}$.

Remark 1.3. - Combining the ideas developed in this paper (and in particular (1.6)) with a global control on the flow given by invariant measures (which itself is related to the Hamiltonian nature of our equation, see e.g. our previous work [8] or [2, 3, 16, 17]) leads to global well posedness results for a class of super critical wave equations (see our forthcoming paper [9]). On the other hand, it would be interesting to decide whether the local in time result of Theorem 1 may be successfully combined with other global controls on the flow such as conservation laws.

Remark 1.4. - The method of proof consists in using the fact that though the initial data have low regularity, their $L^{p}$ properties are (almost surely) much better than expected, allowing the use of a fixed point method after having singled out the linear evolution. Let us note that such $L^{p}$ regularization phenomena are well-known in the context of Fourier series since the work of Paley-Zygmund [13. Similar phenomena were recently studied by Ayache and the second author in the context of sums of type (1.4) in [1].

Remark 1.5. - In the improved time existence statement of Theorem 1 1 one may replace the assumption to deal with Gaussian or Bernoulli's variables by the assumption (3.1) below.

Remark 1.6. - For the sake of conciseness, we chose to focus on the cubic semi linear wave equation. However, the strategy presented here applies to arbitrary non linearities and allows to go beyond the usual critical threshold. 
Finally let us notice that our results extend to Dirichlet or Neumann boundary conditions. In this case, the deterministic Cauchy theory is much less well known.

Definition 1.7. - Let $\left(e_{n}\right)_{n=1}^{\infty}$ be the $L^{2}$-normalized basis consisting in eigenfunctions of the Laplace operator with Dirichlet (resp Neumann) boundary conditions, associated to eigenvalues $\lambda_{n}^{2}$. The space $H_{D}^{s}(M)$ (resp $H_{N}^{s}(M)$ ) is the space of functions $f$ such there exists a sequence $\left(\alpha_{n}\right)_{n=1}^{\infty}$ such that

$$
f(x)=\sum_{n} \alpha_{n} e_{n}(x)
$$

with

$$
\sum_{n}\left(1+\lambda_{n}\right)^{2 s}\left|\alpha_{n}\right|^{2}<\infty
$$

We shall denote by $\mathcal{H}_{D}^{s}(M)=H_{D}^{s}(M) \times H_{D}^{s-1}(M)\left(\operatorname{resp} \mathcal{H}_{N}^{s}=H_{N}^{s}(M) \times H_{N}^{s-1}(M)\right)$.

Remark 1.8. - The space $H_{D}^{s}(M)$ coincides with the usual Sobolev space of order $s$ if $-1 / 2<s<1 / 2$ whereas $H_{N}^{s}(M)$ coincide with the usual Sobolev space of order $s$ if $-3 / 2<s<3 / 2$.

Consider now the wave equation

$$
\left(\partial_{t}^{2}-\boldsymbol{\Delta}\right) u+u^{3}=0,\left.\quad\left(u, \partial_{t} u\right)\right|_{t=0}=\left(f_{1}, f_{2}\right)
$$

with real valued initial data $\left(f_{1}, f_{2}\right) \equiv f \in H^{s}(M) \times H^{s-1}(M) \equiv \mathcal{H}^{s}(M)$, and Dirichlet $\left(\left.u\right|_{\mathbb{R}_{t} \times \partial M}=0\right)$ or Neumann $\left(\left.\frac{\partial u}{\partial n}\right|_{\mathbb{R}_{t} \times \partial M}=0\right)$ boundary conditions.

From the results by Lebeau, Planchon and the first author [7] (see also section 6), one can show that the Cauchy problem is well posed in $\mathcal{H}_{D, N}^{2 / 3}(M)$. Here we show that almost surely, this result can be improved to $s=8 / 21<1 / 2$ ).

Theorem 2. - Assume that $\partial M \neq \emptyset$, and that the random variables have sixth moments uniformly bounded

$$
\exists C>0: \forall n \geq 1, \int_{\Omega}\left(\left|h_{n}(\omega)\right|^{6}+\left|l_{n}(\omega)\right|^{6}\right) d p(\omega)<C .
$$

Let us fix $s \geq \frac{8}{21}$ and $f=\left(f_{1}, f_{2}\right) \in \mathcal{H}_{D, N}^{s}(M)$. Let $f^{\omega} \in L^{2}\left(\Omega ; \mathcal{H}_{D, N}^{s}(M)\right)$ be defined by the randomization (1.3), (1.4). Then there exists $\sigma \geq \frac{2}{3}$ such that for almost all $\omega \in \Omega$ there exist $T_{\omega}>0$ and a unique solution to (1.8) with initial data $f^{\omega}$ in a space continuously embedded in

$$
X_{\omega}=\left(\cos \left(t \sqrt{-\Delta_{D, N}}\right) f_{1}^{\omega}+\frac{\sin \left(t \sqrt{-\Delta_{D, N}}\right) f_{2}^{\omega}}{\sqrt{-\Delta_{D, N}}}\right)+C\left(\left[-T_{\omega}, T_{\omega}\right] ; H_{D, N}^{\sigma}(M)\right) .
$$


More precisely, there exists $C>0, \delta>0$ and for every $0<T \leq 1$, an event $\Omega_{T}$ such that

$$
p\left(\Omega_{T}\right) \geq 1-C T^{1+\delta}
$$

and such that for every $\omega \in \Omega_{T}$ there exists a unique solution $u$ of (1.1) with data $f^{\omega}$ in a space continuously embedded in $C\left([0, T] ; H^{s}(M)\right)$.

Moreover, if $h_{n}, l_{n}$ are standard real Gaussian or Bernoulli variables one can improve (1.10) to

$$
p\left(\Omega_{T}\right) \geq 1-C e^{-c / T^{\delta}}, \quad c, \delta>0 .
$$

The paper is organized as follows: in section 2 we recall Strichartz estimates for wave equations and Sogge's estimates for $L^{p}$ norms of spectral projectors. In Section 3 we prove a large deviation bound. In Section 4 we prove in some sense that "randomization beats deterministic Strichartz estimates" in terms of $L^{p}$ estimates. In Section 5 we perform a classical fixed point argument in a suitable space to prove Theorem 1, Finally, in Section 6 we indicate how the previous argument have to be adapted in the case of a boundary value problem. In all the proof, we shall focus on positive times, the case of negative times being similar due to time reversibility.

Acknowledgments: We thank H. Queffélec for providing us the reference [13].

\section{Strichartz and Sogge estimates}

We shall assume in this section that the boundary of $M$ is empty and collect the Strichartz estimates for the free evolution and the Sogge estimates for the eigenfunctions $e_{n}(x)$. These sets of estimates are actually in the same family, their proofs being a combination of the Fourier integral operator approximation for hyperbolic problems and the $T T^{\star}$ duality argument. Let us note that due to the finite speed of propagation for solutions to the wave equation, Strichartz estimates on a compact manifold are equivalent to some variable coefficient Strichartz estimates on $\mathbb{R}^{3}$. We refer to Kapitanskii [11] for the proof of the Strichartz estimate we state bellow and to Sogge [15] for the bound on $e_{n}$ stated bellow.

The purpose of the next definition is to define the Strichartz spaces used for solving the problem (1.1).

Definition 2.1. - For $0 \leq s<1$, a couple of real numbers $(p, q), \frac{2}{s} \leq p \leq+\infty$ is $s$-admissible if

$$
\frac{1}{p}+\frac{3}{q}=\frac{3}{2}-s
$$


For $T>0,0 \leq s<1$, we define the spaces

$$
X_{T}^{s}=C^{0}\left([0, T] ; H^{s}(M)\right) \underset{(p, q) \text { s- admissible }}{ } L^{p}\left((0, T) ; L^{q}(M)\right)
$$

and its dual space

$$
Y_{T}^{s}=L^{1}\left([0, T] ; H^{-s}(M)\right)+{ }_{(p, q)} s \text { - admissible } L^{p^{\prime}}\left((0, T) ; L^{q^{\prime}}(M)\right)
$$

$\left(p^{\prime}, q^{\prime}\right)$ being the conjugate couple of $(p, q)$, equipped with their natural norms (notice that to define these spaces, we keep only the extremal couples corresponding to $p=2 / s$ and $p=+\infty$ respectively).

We next state the Strichartz inequality for the wave equation, posed on a three dimensional smooth compact (without boundary) riemannian manifold.

Proposition 2.2. - Let $(p, q)$ be an s-admissible couple. Then there exists $C>0$ such that for every $T \in] 0,1]$, every $f \in H^{s}(M)$ one has

$$
\left\|e^{ \pm i t \sqrt{-\boldsymbol{\Delta}}}(f)\right\|_{L^{p}\left([-T, T] ; L^{q}(M)\right)} \leq C\|f\|_{H^{s}(M)} .
$$

Let us now state a corollary of Proposition 2.2 .

Corollary 2.3. - For every $0<s<1$, every $s$-admissible couple $(p, q)$, there exists $C>0$ such that for every $T \in] 0,1]$, every $f \in \mathcal{H}^{s}(M), g \in Y_{T}^{1-s}$ one has

$$
\begin{gathered}
\left\|\cos (t \sqrt{-\boldsymbol{\Delta}})\left(f_{1}\right)\right\|_{X_{T}^{s}}+\left\|\frac{\sin (t \sqrt{-\boldsymbol{\Delta}})}{\sqrt{-\boldsymbol{\Delta}}}\left(f_{2}\right)\right\|_{X_{T}^{s}} \leq C\|f\|_{\mathcal{H}^{s}(M)}, \\
\left\|\int_{0}^{t} \frac{\sin ((t-\tau) \sqrt{-\boldsymbol{\Delta}})}{\sqrt{-\boldsymbol{\Delta}}}(g)(\tau) d \tau\right\|_{X_{T}^{s}} \leq C\|g\|_{Y_{T}^{1-s}}
\end{gathered}
$$

The proof of Corollary 2.3 can essentially be found in [8]. Notice that here we have to modify slightly the argument to take care of the 0 eigenvalue of the Laplace operator. We next state the Sogge estimate which will be involved in our analysis.

Proposition 2.4. - There exists $C>0$ such that for every $n \geq 1$,

$$
\left\|e_{n}\right\|_{L^{4}(M)} \leq C\left(1+\lambda_{n}^{2}\right)^{\frac{1}{8}} .
$$

Let us note that the $L^{4}(M)$ norm can be replaced by other $L^{p}(M), 2 \leq p \leq \infty$ norms by modifying appropriately the power of $1+\lambda_{n}^{2}$ according to an interpolation with the trivial $L^{2}$ bound or the $L^{\infty}$ Weyl bound. We also note that the estimate of Proposition 2.4 has a natural extension to other dimensions, the index 4 being replaced by $2(d+1) /(d-1)$. Finally, the estimate for $e_{n}$ given by Proposition 2.4 also holds, with the appropriate statement, for the spectral projection on $\sqrt{-\boldsymbol{\Delta}} \in[\lambda, \lambda+1]$. 


\section{A large deviation bound}

The purpose of this section is to prove the following statement.

Lemma 3.1. - Let $\left(l_{n}(\omega)\right)_{n=1}^{\infty}$ be a sequence of real, independent random variables with associated sequence of distributions $\left(\mu_{n}\right)_{n=1}^{\infty}$. Assume that $\mu_{n}$ satisfy the property

$$
\exists c>0: \forall \gamma \in \mathbb{R}, \forall n \geq 1,\left|\int_{-\infty}^{\infty} e^{\gamma x} d \mu_{n}(x)\right| \leq e^{c \gamma^{2}} .
$$

Then there exists $\alpha>0$ such that for every $\lambda>0$, every sequence $\left(c_{n}\right)_{n=1}^{\infty} \in l^{2}$ of real numbers,

$$
p\left(\omega:\left|\sum_{n=1}^{\infty} c_{n} l_{n}(\omega)\right|>\lambda\right) \leq 2 e^{-\frac{\alpha \lambda^{2}}{\sum_{n} c_{n}^{2}}} .
$$

As a consequence there exists $C>0$ such that for every $p \geq 2$, every $\left(c_{n}\right)_{n=1}^{\infty} \in l^{2}$,

$$
\left\|\sum_{n=1}^{\infty} c_{n} l_{n}(\omega)\right\|_{L^{p}(\Omega)} \leq C \sqrt{p}\left(\sum_{n=1}^{\infty} c_{n}^{2}\right)^{1 / 2} .
$$

Remark 3.2. - Let us notice that (3.1) is readily satisfied if $\left(l_{n}(\omega)\right)_{n=1}^{\infty}$ are standard real Gaussian or standard Bernoulli variables. Indeed in the case of Gaussian

$$
\int_{-\infty}^{\infty} e^{\gamma x} d \mu_{n}(x)=\int_{-\infty}^{\infty} e^{\gamma x} e^{-x^{2} / 2} \frac{d x}{\sqrt{2 \pi}}=e^{\gamma^{2} / 2}
$$

In the case of Bernoulli variables (or more generally any random variables having compactly supported distribution) one can obtain that (3.1) is satisfied by invoking the inequality

$$
\frac{e^{\gamma}+e^{-\gamma}}{2} \leq e^{\gamma^{2} / 2}, \quad \forall \gamma \in \mathbb{R}
$$

Proof of Lemma 3.1. - We give an argument similar to the proof of [18, Lemma 4.2]. In the case of Gaussian we can see Lemma 3.1 as a very particular case of a $L^{p}$ smoothing properties of the Hartree-Foch heat flow (see e.g. [18, Section 3] for more details on this issue). For $t>0$ to be determined later, using the independence and (3.1), we obtain

$$
\begin{aligned}
& \int_{\Omega} e^{t \sum_{n \geq 1} c_{n} l_{n}(\omega)} d p(\omega)=\prod_{n \geq 1} \int_{\Omega} e^{t c_{n} l_{n}(\omega)} d p(\omega) \\
&=\prod_{n \geq 1} \int_{-\infty}^{\infty} e^{t c_{n} x} d \mu_{n}(x) \leq \prod_{n \geq 1} e^{c\left(t c_{n}\right)^{2}}=e^{\left(c t^{2}\right) \sum_{n} c_{n}^{2}} .
\end{aligned}
$$


Therefore

$$
e^{\left(c t^{2}\right) \sum_{n} c_{n}^{2}} \geq e^{t \lambda} p\left(\omega: \sum_{n \geq 1} c_{n} l_{n}(\omega)>\lambda\right)
$$

or equivalently,

$$
p\left(\omega: \sum_{n \geq 1} c_{n} l_{n}(\omega)>\lambda\right) \leq e^{\left(c t^{2}\right) \sum_{n} c_{n}^{2}} e^{-t \lambda} .
$$

We choose $t$ as

$$
t \equiv \frac{\lambda}{2 c \sum_{n} c_{n}^{2}}
$$

Hence

$$
p\left(\omega: \sum_{n \geq 1} c_{n} l_{n}(\omega)>\lambda\right) \leq e^{-\frac{\lambda^{2}}{4 c \sum_{n} c_{n}^{2}}} .
$$

In the same way (replacing $c_{n}$ by $-c_{n}$ ), we can show that

$$
p\left(\omega: \sum_{n \geq 1} c_{n} l_{n}(\omega)<-\lambda\right) \leq e^{-\frac{\lambda^{2}}{4 c \sum_{n} c_{n}^{2}}}
$$

which completes the proof of (3.2). To deduce (3.3), we write

$$
\begin{aligned}
& \left\|\sum_{n=1}^{\infty} c_{n} l_{n}(\omega)\right\|_{L^{p}(\Omega)}^{p}=p \int_{0}^{+\infty} p\left(\omega:\left|\sum_{n=1}^{\infty} c_{n} l_{n}(\omega)\right|>\lambda\right) \lambda^{p-1} d \lambda \\
& \quad \leq C p \int_{0}^{+\infty} \lambda^{p-1} e^{-\frac{c \lambda^{2}}{\sum_{n} c_{n}^{2}}} d \lambda \leq C p\left(C \sum_{n} c_{n}^{2}\right)^{\frac{p}{2}} \int_{0}^{+\infty} \lambda^{p-1} e^{-\frac{\lambda^{2}}{2}} d \lambda \leq C\left(C p \sum_{n} c_{n}^{2}\right)^{\frac{p}{2}}
\end{aligned}
$$

which completes the proof of Lemma 3.1 .

\section{Averaging effects}

In this section, we exploit the randomization to get two $L^{4}$ estimates for the free evolution. These estimates play a central role in the proof of Theorem 1 .

Proposition 4.1. - Let $s \geq 1 / 4$ and $0<T \leq 1$. Under the assumptions of Theorem 1 , for $f=\left(f_{1}, f_{2}\right) \in \mathcal{H}^{s}(M)$, we consider the free evolution with data $f^{\omega}$, given by

$$
u_{f}^{\omega}(t, x)=\cos (t \sqrt{-\boldsymbol{\Delta}}) f_{1}^{\omega}+\frac{\sin (t \sqrt{-\boldsymbol{\Delta}})}{\sqrt{-\boldsymbol{\Delta}}} f_{2}^{\omega} .
$$

Then there exists $C>0$ such that for every $f \in \mathcal{H}^{s}(M)$,

$$
\left\|(-\boldsymbol{\Delta}+1)^{\frac{s}{2}-\frac{1}{8}} u_{f}^{\omega}\right\|_{L^{4}\left(\Omega_{\omega} \times[0, T]_{t} \times M_{x}\right)} \leq C T^{1 / 4}\|f\|_{\mathcal{H}^{s}(M)} .
$$


In particular, thanks to the Bienaymé-Tchebichev inequality, if we set

$$
E_{\lambda, T, f}=\left\{\omega \in \Omega:\left\|(-\boldsymbol{\Delta}+1)^{\frac{s}{2}-\frac{1}{8}} u_{f}^{\omega}\right\|_{L^{4}\left([0, T]_{t} \times M_{x}\right)} \geq \lambda\right\}
$$

then there exists $C>0$ such that for every $\lambda>0$, every $f \in \mathcal{H}^{s}(M)$,

$$
p\left(E_{\lambda, T, f}\right) \leq C T \lambda^{-4}\|f\|_{\mathcal{H}^{s}(M)}^{4} .
$$

Proof. - By expanding the sines and cosines functions as sums of exponentials, we obtain that we may only consider the contribution of $(-\boldsymbol{\Delta}+1)^{\frac{s}{2}-\frac{1}{8}} e^{i t \sqrt{-\boldsymbol{\Delta}}} f_{1}^{\omega}$, the other contributions being dealt with similarly (again the zero frequency should be treated separately). Suppose that

$$
f_{1}(x)=\sum_{n} \alpha_{n} e_{n}(x) \in H^{s}(M) .
$$

If we set $\widetilde{\alpha}_{n}=\left(\lambda_{n}^{2}+1\right)^{\frac{s}{2}-\frac{1}{8}} \alpha_{n}$ then

$$
\left\|f_{1}\right\|_{H^{s}(M)}^{2}=\sum_{n}\left|\widetilde{\alpha}_{n}\right|^{2}\left(1+\lambda_{n}^{2}\right)^{\frac{1}{4}} .
$$

We shall use the following result.

Lemma 4.2. - Assume that $\left(h_{n}\right)_{n=1}^{\infty}$ is a sequence of independent, 0-mean value, complex random variables satisfying for some $k \in \mathbb{N}^{*}$

$$
\exists C>0, \forall n \geq 1, \int_{\Omega}\left|h_{n}(\omega)\right|^{2 k} d p(\omega) \leq C
$$

then

$$
\begin{gathered}
\forall 2 \leq p \leq 2 k, \quad \exists C>0, \quad \forall\left(c_{n}\right)_{n \in \mathbb{N}^{*}} \in l^{2}\left(\mathbb{N}^{*}, \mathbb{C}\right), \\
\left\|\sum_{n} c_{n} h_{n}\right\|_{L^{p}(\Omega)} \leq C\left(\sum_{n}\left|c_{n}\right|^{2}\right)^{1 / 2} .
\end{gathered}
$$

Proof. - Using Hölder inequality, it suffices to prove the estimate for $p=2 k$. We have

$$
\begin{aligned}
\int_{\Omega}\left|\sum_{n} c_{n} h_{n}(\omega)\right|^{2 k}=\sum_{n_{1}, \cdots, n_{2 k}} \int_{\Omega} c_{n_{1}} \times \cdots \times c_{n_{k}} \overline{c_{n_{k+1}} \times \cdots \times c_{n_{2 k}}} \\
h_{n_{1}}(\omega) \times \cdots \times h_{n_{k}}(\omega) \overline{h_{n_{k+1}}(\omega) \times \cdots \times h_{n_{2 k}}(\omega)} d p(\omega) .
\end{aligned}
$$

Using the independence and the fact that the random variables have 0 mean, we obtain that for the contribution of $n_{1}, \cdots, n_{2 k}$ not to vanish, each index have to be appear at 
least twice. As a consequence (using that the $2 k$-th moment of the random variables are uniformly bounded)

$$
\begin{aligned}
\int_{\Omega}\left|\sum_{n} c_{n} h_{n}(\omega)\right|^{2 k} & \leq \sum_{n_{1}, \cdots, n_{k}} \int_{\Omega}\left|c_{n_{1}} \cdots c_{n_{k}}\right|^{2}\left|h_{n_{1}}(\omega) \cdots h_{n_{k}}(\omega)\right|^{2} d p(\omega) \\
& \leq C\left(\sum_{n}\left|c_{n}\right|^{2}\right)^{k}
\end{aligned}
$$

Let us come back to the proof of Proposition 4.1, Using Lemma 4.2, we obtain

$$
\begin{aligned}
\left\|(-\boldsymbol{\Delta}+1)^{\frac{s}{2}-\frac{1}{8}} e^{i t \sqrt{-\Delta}} f_{1}^{\omega}\right\|_{L^{4}\left(\Omega_{\omega} \times[0, T]_{t} \times M_{x}\right)} & \leq C\left\|\left(\sum_{n}\left|\widetilde{\alpha}_{n} e_{n}(x)\right|^{2}\right)^{1 / 2}\right\|_{L^{4}((0, T) \times M)} \\
& =C\left\|\sum_{n}\left|\widetilde{\alpha}_{n} e_{n}(x)\right|^{2}\right\|_{L^{2}((0, T) \times M)}^{1 / 2} \\
& \leq C\left(\sum_{n}\left\|\left|\widetilde{\alpha}_{n} e_{n}(x)\right|^{2}\right\|_{L^{2}((0, T) \times M)}\right)^{1 / 2} \\
& \leq C T^{1 / 4}\left(\sum_{n}\left\|\widetilde{\alpha}_{n} e_{n}(x)\right\|_{L^{4}(M)}^{2}\right)^{1 / 2}
\end{aligned}
$$

which, according to Proposition 2.4 implies Proposition 4.1,

Remark 4.3. - For $1<p<+\infty$, the norm $\|f\|_{W^{s, p}(M)}$ and $\left\|(-\boldsymbol{\Delta}+1)^{\frac{s}{2}}\right\|_{L^{p}(M)}$ are equivalent. Indeed, for $s \in 2 \mathbb{N}$, this is a consequence of the $L^{p}$ elliptic regularity theorem and for general $s$ it follows by interpolation and duality.

As a consequence of Lemma 3.1, under assumption (3.1), we can improve the averaging effect estimate as follows.

Proposition 4.4. - Under the assumption of Proposition 4.1, if we suppose that in addition the randomization obeys the condition (3.1), then for $p \geq 4$,

$$
\left\|(-\boldsymbol{\Delta}+1)^{\frac{s}{2}-\frac{1}{8}} u_{f}^{\omega}\right\|_{L^{p}\left(\Omega ; L^{4}\left([0,1]_{t} \times M_{x}\right)\right)} \leq C p^{\frac{1}{2}}\|f\|_{\mathcal{H}^{s}(M)} .
$$

As a consequence, if we set

$$
E_{\lambda, f}=\left\{\omega \in \Omega:\left\|(-\boldsymbol{\Delta}+1)^{\frac{s}{2}-\frac{1}{8}} u_{f}^{\omega}\right\|_{L^{4}\left([0,1]_{t} \times M_{x}\right)} \geq \lambda\right\}
$$

then there exist $C>0$ and $c>0$ such that for every $\lambda>0$, every $f \in \mathcal{H}^{s}(M)$,

$$
p\left(E_{\lambda, f}\right) \leq C e^{-c \lambda^{2} /\|f\|_{\mathcal{H}^{s}(M)}^{2}} .
$$


Proof. - As in the proof of Proposition 4.1, we may only consider the contribution of $(-\boldsymbol{\Delta}+1)^{\frac{s}{2}-\frac{1}{8}} e^{i t \sqrt{-\boldsymbol{\Delta}}} f_{1}^{\omega}$. Writing $f_{1}=\sum_{n} \alpha_{n} e_{n}$ and if we set $\widetilde{\alpha}_{n}=\left(\lambda_{n}^{2}+1\right)^{\frac{s}{2}-\frac{1}{8}} \alpha_{n}$ then

$$
\left\|f_{1}\right\|_{H^{s}(M)}^{2}=\sum_{n}\left|\widetilde{\alpha}_{n}\right|^{2}\left(1+\lambda_{n}^{2}\right)^{\frac{1}{4}}
$$

Set

$$
v_{f_{1}}^{\omega}(t, x) \equiv(-\boldsymbol{\Delta}+1)^{\frac{s}{2}-\frac{1}{8}} e^{i t \sqrt{-\Delta}} f_{1}^{\omega} .
$$

Thus the issue is to show that

$$
\left\|v_{f_{1}}^{\omega}(t, x)\right\|_{L^{p}\left(\Omega ; L^{4}\left([0,1]_{t} \times M_{x}\right)\right)} \leq C \sqrt{p}\left\|f_{1}\right\|_{H^{s}(M)} .
$$

By the Minkowski inequality, for $p \geq 4$,

$$
\left\|v_{f_{1}}^{\omega}(t, x)\right\|_{L^{p}\left(\Omega ; L^{4}\left([0,1]_{t} \times M_{x}\right)\right)} \leq\left\|v_{f_{1}}^{\omega}(t, x)\right\|_{L^{4}\left([0,1]_{t} \times M_{x} ; L^{p}(\Omega)\right)} .
$$

Thanks to Lemma 3.1,

$$
\left\|v_{f_{1}}^{\omega}(t, x)\right\|_{L^{p}(\Omega)}=\left\|\sum_{n} \widetilde{\alpha}_{n} e^{i t \lambda_{n}} e_{n}(x) h_{n}(\omega)\right\|_{L^{p}(\Omega)} \leq C \sqrt{p}\left(\sum_{n}\left|\widetilde{\alpha_{n}} e_{n}(x)\right|^{2}\right)^{1 / 2} .
$$

Therefore, we get, thanks to Proposition 2.4.

$$
\begin{aligned}
\left\|v_{f_{1}}^{\omega}(t, x)\right\|_{L^{p}\left(\Omega ; L^{4}\left([0,1]_{t} \times M_{x}\right)\right)} & \leq C \sqrt{p}\left\|\left(\sum_{n}\left|\widetilde{\alpha_{n}} e_{n}(x)\right|^{2}\right)^{1 / 2}\right\|_{L^{4}\left([0,1]_{t} \times M_{x}\right)} \\
& \leq C \sqrt{p}\left(\left\|\sum_{n}\left|\widetilde{\alpha_{n}} e_{n}(x)\right|^{2}\right\|_{L^{2}\left(M_{x}\right)}\right)^{1 / 2} \\
& \leq C \sqrt{p}\left(\sum_{n}\left\|\left.\widetilde{\alpha_{n}} e_{n}(x)\right|^{2}\right\|_{L^{2}\left(M_{x}\right)}\right)^{1 / 2} \\
& \leq C \sqrt{p}\left(\sum_{n}\left|\widetilde{\alpha_{n}}\right|^{2}\left\|e_{n}(x)\right\|_{L^{4}\left(M_{x}\right)}^{2}\right)^{1 / 2} \\
& \leq C \sqrt{p}\left(\sum_{n}\left|\widetilde{\alpha_{n}}\right|^{2}\left(1+\lambda_{n}^{2}\right)^{1 / 4}\right)^{1 / 2}
\end{aligned}
$$

which completes the proof of (4.3). Let us now turn to the proof of (4.4). Thanks to the Bienaymé-Tchebichev inequality, there exists $\alpha>0$ such that for every $p \geq 4$, every $f \in \mathcal{H}^{s}(M)$,

$$
p\left(E_{\lambda, f}\right) \leq \lambda^{-p}\left(\alpha \sqrt{p}\|f\|_{\mathcal{H}^{s}(M)}\right)^{p} .
$$

Inequality (4.4) easily holds, if $\lambda$ is such that

$$
\frac{\lambda}{\|f\|_{\mathcal{H}^{s}(M)}} \leq 2 \alpha e
$$


If (4.6) does not hold, we set

$$
p \equiv\left[\frac{\lambda}{\alpha\|f\|_{\mathcal{H}^{s}(M)}^{e}}\right]^{2}(\geq 4)
$$

With this choice of $p$, we come back to (4.5) which yields (4.4). This completes the proof of Proposition 4.4 .

\section{The fixed point}

If we wish to solve

$$
\left(\partial_{t}^{2}-\boldsymbol{\Delta}\right) u+u^{3}=0,\left.\quad\left(u, \partial_{t} u\right)\right|_{t=0}=\left(f_{1}^{\omega}, f_{2}^{\omega}\right)=f^{\omega}
$$

by writing $u=u_{f}^{\omega}+v$, where $u_{f}^{\omega}$ denotes the free evolution associated to $f^{\omega}$, we obtain that $v$ solves

$$
\left(\partial_{t}^{2}-\boldsymbol{\Delta}\right) v=-\left(u_{f}^{\omega}+v\right)^{3},\left.\quad\left(v, \partial_{t} v\right)\right|_{t=0}=(0,0) .
$$

Write this equation as

$$
v(t, \cdot)=-\int_{0}^{t} \frac{\sin ((t-\tau) \sqrt{-\boldsymbol{\Delta}})}{\sqrt{-\boldsymbol{\Delta}}}\left(\left(u_{f}^{\omega}+v\right)^{3}\right)(\tau, \cdot) d \tau .
$$

Define the map

$$
K_{f}^{\omega}: v \longmapsto-\int_{0}^{t} \frac{\sin ((t-\tau) \sqrt{-\Delta})}{\sqrt{-\boldsymbol{\Delta}}}\left(\left(u_{f}^{\omega}+v\right)^{3}\right)(\tau, \cdot) d \tau .
$$

5.1. The case $s=1 / 4$. - In this case, the numerology is particularly simple

Proposition 5.1. - Let us fix $s=1 / 4$. Then there exists $C>0$ such that for every $T \in[0,1]$, every $f \in \mathcal{H}^{1 / 4}(M)$, every $\lambda>0$ for $\omega \in E_{\lambda, f}^{c}$ the map $K_{f}^{\omega}$ satisfies

$$
\begin{gathered}
\left\|K_{f}^{\omega}(v)\right\|_{X_{T}^{1 / 2}} \leq C\left(\lambda^{3}+\|v\|_{X_{T}^{1 / 2}}^{3}\right), \\
\left\|K_{f}^{\omega}(v)-K_{f}^{\omega}(w)\right\|_{X_{T}^{1 / 2}} \leq C\|v-w\|_{X_{T}^{1 / 2}}\left(\lambda^{2}+\|v\|_{X_{T}^{1 / 2}}^{2}+\|w\|_{X_{T}^{1 / 2}}^{2}\right) .
\end{gathered}
$$

Proof. - Indeed, for $\omega \in E_{\lambda, f}^{c}$, we have

$$
\left\|u_{f}^{\omega}\right\|_{L^{4}((0, T) \times M)} \leq \lambda
$$

and consequently, according to Corollary 2.3 ,

$$
\left\|K_{f}^{\omega}(v)\right\|_{X_{T}^{1 / 2}} \leq C\left\|\left(u_{f}^{\omega}+v\right)^{3}\right\|_{L^{4 / 3}([0, T] \times M)} \leq C\left(\lambda^{3}+\|v\|_{X_{T}^{1 / 2}}^{3}\right)
$$


and

$$
\begin{aligned}
& \left\|K_{f}^{\omega}(v)-K_{f}^{\omega}(w)\right\|_{X_{T}^{1 / 2}} \\
& \quad \leq C\left\|\left(u_{f}^{\omega}+v\right)^{3}-\left(u_{f}^{\omega}+w\right)^{3}\right\|_{L^{4 / 3}([0, T] \times M)} \leq C\|v-w\|_{X_{T}^{1 / 2}}\left(\lambda^{2}+\|v\|_{X_{T}^{1 / 2}}^{2}+\|v\|_{X_{T}^{1 / 2}}^{2}\right)
\end{aligned}
$$

\subsection{The case $s>1 / 4$. -}

Proposition 5.2. - Let us fix $s>1 / 4$. Then there exists $\sigma>1 / 2, C>0$ and $\kappa>0$ such that for every $T \in[0,1]$, every $f \in H^{s}(M)$, every $\lambda>0, \omega \in E_{\lambda, f}^{c}$ the map $K_{f}^{\omega}$ satisfies

$$
\begin{gathered}
\left\|K_{f}^{\omega}(v)\right\|_{X_{T}^{\sigma}} \leq C\left(\lambda^{3}+T^{\kappa}\|v\|_{X_{T}^{\sigma}}^{3}\right), \\
\left\|K_{f}^{\omega}(v)-K_{f}^{\omega}(w)\right\|_{X_{T}^{\sigma}} \leq C T^{\kappa}\|v-w\|_{X_{T}^{\sigma}}\left(\lambda^{2}+\|v\|_{X_{T}^{\sigma}}^{2}+\|w\|_{X_{T}^{\sigma}}^{2}\right) .
\end{gathered}
$$

Proof. - Let us notice that, according to Corollary 2.3, for $1 / 2<\sigma<1$ (to be fixed later),

where

$$
\left\|K_{f}^{\omega}(v)\right\|_{X_{T}^{\sigma}} \leq C\left\|\left(u_{f}+v\right)^{3}\right\|_{L^{p^{\prime}}\left([0, T] ; L^{q^{\prime}}(M)\right)},
$$

$$
\frac{1}{p}+\frac{3}{q}=\frac{3}{2}-(1-\sigma), \quad p=4 .
$$

Notice that $4>2 / \sigma$ and thus the choice $p=4$ is allowed. Using the triangle inequality, we obtain

$$
\left\|K_{f}^{\omega}(v)\right\|_{X_{T}^{\sigma}} \leq C\left(\left\|u_{f}^{\omega}\right\|_{L^{4}\left([0, T] ; L^{\left.3 q^{\prime}(M)\right)}\right.}^{3}+\|v\|_{L^{4}\left([0, T] ; L^{\left.3 q^{\prime}(M)\right)}\right.}^{3}\right) .
$$

Let us first study the second term in the right hand-side of (5.6). This will be done by invoking the $H^{\sigma}, \sigma>1 / 2$ well-posedness argument. Observe that $\frac{1}{q^{\prime}}=\frac{11}{12}-\frac{\sigma}{3}$. Let $p$ be such that $\left(p, 3 q^{\prime}\right)$ is $\sigma$-admissible, i.e.

$$
\frac{1}{p}+\frac{3}{3 q^{\prime}}=\frac{3}{2}-\sigma, \quad \Rightarrow \quad \frac{1}{p}=\frac{7}{12}-\frac{2 \sigma}{3} .
$$

Observe that since $\sigma>1 / 2$ we have that $p>4$. Therefore thanks to the Hölder inequality (in time) for $\sigma>1 / 2$ there exists $\kappa>0$ such that

$$
\|v\|_{L^{4}\left([0, T] ; L^{\left.3 q^{\prime}(M)\right)}\right.}^{3} \leq T^{\kappa}\|v\|_{X_{T}^{\sigma}}^{3} .
$$

Let us next study the first term in the right hand-side of (5.6). We first consider the case $s \geq 1$. Using the Sobolev inequality, for $\omega \in E_{\lambda, f}^{c}$, we can write

$$
\left\|u_{f}^{\omega}\right\|_{L^{4}([0,1]) ; L^{\left.3 q^{\prime}(M)\right)}} \leq C\left\|u_{f}^{\omega}\right\|_{L^{4}\left([0,1] ; W^{s-\frac{1}{4}, 4}(M)\right)} \leq C \lambda .
$$


This ends the proof of (5.4) for $s \geq 1$ ( $\sigma$ being an arbitrary number in $(1 / 2,1)$ ).

Let us next assume that $s<1$. Then for $\omega \in E_{\lambda, f}^{c}$ (and according to Sobolev embedding), we have

$$
\left\|u_{f}^{\omega}\right\|_{\left.L^{4}([0,1]) ; L^{q_{0}}(M)\right)} \leq C\left\|u_{f}^{\omega}\right\|_{L^{4}\left([0,1] ; W^{s-\frac{1}{4}, 4}(M)\right)} \leq C \lambda
$$

where

$$
\frac{1}{q_{0}}=\frac{1}{4}-\frac{(s-1 / 4)}{3}
$$

We choose

$$
\sigma=\min \left(\frac{9}{10}, \frac{1}{2}+3\left(s-\frac{1}{4}\right)\right)
$$

which fixes the value of $\sigma$ in the case $s<1$. Then

$$
\frac{1}{4}+\frac{3}{q}=\frac{3}{2}-(1-\sigma) \Rightarrow \frac{1}{q}=\frac{1}{4}-\frac{(1 / 2-\sigma)}{3} \Rightarrow \frac{1}{3 q^{\prime}} \geq \frac{1}{4}-\frac{(s-1 / 4)}{3}=\frac{1}{q_{0}} .
$$

As a consequence for $\omega \in E_{\lambda, f}^{c}$, using the Hölder inequality in space, we get that for $s<1$, we can bound the contribution of the first term in the right hand-side of (5.6) as follows

$$
\left\|u_{f}^{\omega}\right\|_{L^{4}([0,1]) ; L^{\left.3 q^{\prime}(M)\right)}} \leq\left\|u_{f}^{\omega}\right\|_{\left.L^{4}([0,1]) ; L^{q_{0}}(M)\right)} \leq C\left\|u_{f}^{\omega}\right\|_{L^{4}\left([0,1] ; W^{s-\frac{1}{4}, 4}(M)\right)} \leq C \lambda .
$$

This ends the proof of (5.4). The proof of (5.5) is similar. Indeed, it suffices to write

$$
\begin{aligned}
&\left\|K_{f}^{\omega}(v)-K_{f}^{\omega}(w)\right\|_{X_{T}^{\sigma}} \leq\|v-w\|_{L^{4}\left([0, T] ; L^{3 q^{\prime}}(M)\right)}\left(\left\|u_{f}^{\omega}\right\|_{L^{4}\left([0, T] ; L^{3 q^{\prime}}(M)\right)}^{2}\right. \\
&\left.+\|v\|_{L^{4}\left([0, T] ; L^{\left.3 q^{\prime}(M)\right)}\right.}^{2}+\|w\|_{L^{4}\left([0, T] ; L^{\left.3 q^{\prime}(M)\right)}\right.}^{2}\right)
\end{aligned}
$$

and to use the previous estimates. This completes the proof of Proposition 5.2 ,

Let us now complete the proof of Theorem 1, Let us first consider the case of a randomization induced by a general family of random variables satisfying (1.2). Fix $0<T \leq 1$. As a consequence of Propositions 5.1 and 5.2, if $\omega \in E_{\lambda, T, f}^{c}$ and if

$$
C \lambda^{3}+T^{\kappa}\left(2 C \lambda^{3}\right)^{3} \leq 2 C \lambda^{3}, \text { and } C T^{\kappa}\left(\lambda^{2}+\lambda^{6}\right) \leq \frac{1}{2}, \quad \kappa \geq 0,(\kappa>0 \text { if } s>1 / 4),
$$

then the map $K_{f}^{\omega}$ is a contraction on the ball of radius $2 C \lambda^{3}$ of $X_{T}^{\sigma}$. Notice that the condition (5.7) above is implied by the following

$$
T^{\kappa} \lambda^{6}=\epsilon^{6} \ll 1
$$

As a consequence, if we define, with $\delta=\kappa / 6$,

$$
\Omega_{T}=E_{\lambda=\epsilon T^{-\delta, T, f}}^{c}, \quad \Sigma=\bigcup_{n \in \mathbb{N}^{*}} \Omega_{1 / n},
$$


then

$$
p\left(\Omega_{T}\right) \geq 1-C T^{1+4 \delta}, \quad p(\Sigma)=1
$$

and we obtain the first part in Theorem 1 in the case of general variables satisfying only (1.2).

Let us finally consider the case of random variables satisfying in addition to (1.2) the property (3.1). In this case $\lambda=\lambda(T)$ is chosen such that

$$
T^{\kappa} \lambda^{6}=\epsilon \ll 1
$$

and according to Proposition [5.2, if $\omega \in E_{\lambda, T, f}^{c}$, then the map $K_{f}^{\omega}$ is a contraction on the ball of radius $2 C \lambda^{3}$ of $X_{T}^{\sigma}$. Now according to (4.4), we obtain that if we set

$$
\Omega_{T}=E_{\lambda(T), f}^{c}, \quad \Sigma=\bigcup_{n \in \mathbb{N}^{*}} \Omega_{1 / n}
$$

then

$$
p\left(\Omega_{T}\right) \geq 1-C e^{-c / T^{\delta}}, \delta, C, c>0, \quad p(\Sigma)=1 .
$$

This completes the proof of Theorem 1 .

Remark 5.3. - Let us observe that in Theorem 1 if $s>1 / 4$ then $\sigma>1 / 2$.

\section{Manifolds with boundary}

In this section we consider the case of Dirichlet or Neumann boundary conditions. For conciseness, we shall drop the subscript $D, N$.

6.1. Strichartz and spectral projector estimates. - The following spectral projector estimate is proved by Smith and Sogge [14]

Proposition 6.1. - There exists $C>0$ such that for every $n \geq 1$,

$$
\left\|e_{n}\right\|_{L^{5}(M)} \leq C\left(1+\lambda_{n}^{2}\right)^{\frac{1}{5}} .
$$

This estimate implies (see [7, Theorem 2]) the following Strichartz inequality

Proposition 6.2. - There exists $C>0$ such that for

$$
\left\|e^{ \pm i t \sqrt{-\Delta}} f\right\|_{L^{5}((0,1) \times M)} \leq C\|f\|_{H \frac{7}{10}(M)} .
$$

By interpolation and duality, we deduce that the Strichartz inequalities (2.4) and (2.5) remain true provided we replace the definition of admissible couples by 
Definition 6.3. - Let $0 \leq s \leq 1$. A couple $(p, q)$ is $s$-admissible if

$$
\frac{1}{p}+\frac{3}{q}=\frac{3}{2}-s
$$

and

$$
p \geq\left\{\begin{array}{l}
\frac{7}{2 s} \text { if } s \leq \frac{7}{10}, \\
5 \text { if } s \geq \frac{7}{10}
\end{array}\right.
$$

\subsection{Averaging effects. -}

Proposition 6.4. - Let $s \geq \frac{2}{5}$. Under the assumptions of Theorem 圆, for $f=\left(f_{1}, f_{2}\right) \in$ $\mathcal{H}^{s}(M)$, we consider the free evolution with data $f^{\omega}$, given by

$$
u_{f}^{\omega}(t, x)=\cos (t \sqrt{-\boldsymbol{\Delta}}) f_{1}^{\omega}+\frac{\sin (t \sqrt{-\boldsymbol{\Delta}}) f_{2}^{\omega}}{\sqrt{-\boldsymbol{\Delta}}} .
$$

Then there exists $C>0$ such that for every $f \in \mathcal{H}^{s}(M)$,

$$
\left\|(-\Delta+1)^{\frac{s}{2}-\frac{1}{5}} u_{f}^{\omega}\right\|_{L^{5}\left(\Omega_{\omega} \times[0, T]_{t} \times M_{x}\right)} \leq C T^{1 / 5}\|f\|_{\mathcal{H}^{s}(M)} \cdot
$$

In particular, thanks to the Bienaymé-Tchebichev inequality, if we set

$$
E_{\lambda, T, f}=\left\{\omega \in \Omega:\left\|(-\boldsymbol{\Delta}+1)^{\frac{s}{2}-\frac{1}{5}} u_{f}^{\omega}\right\|_{L^{5}\left([0, T]_{t} \times M_{x}\right)} \geq \lambda\right\}
$$

then there exists $C>0$ such that for every $\lambda>0$, every $f \in \mathcal{H}^{s}(M)$,

$$
p\left(E_{\lambda, T, f}\right) \leq C T \lambda^{-5}\|f\|_{\mathcal{H}^{s}(M)}^{5} .
$$

Proof. — Using Lemma 4.2 we compute

$$
\begin{aligned}
\left\|(-\boldsymbol{\Delta}+1)^{\frac{s}{2}-\frac{1}{5}} e^{i t \sqrt{-\Delta}} f_{1}^{\omega}\right\|_{L^{5}\left(\Omega_{\omega} \times[0, T]_{t} \times M_{x}\right)} & =\left\|\sum_{n} e^{i t \lambda_{n}}\left(1+\lambda_{n}^{2}\right)^{\frac{s}{2}-\frac{1}{5}} \alpha_{n} e_{n}(x) h_{n}(\omega)\right\|_{L^{5}\left(\Omega_{\omega} \times[0, T]_{t} \times M_{x}\right)} \\
& \leq C\left\|\left(\sum_{n}\left|e^{i t \lambda_{n}}\left(1+\lambda_{n}^{2}\right)^{\frac{s}{2}-\frac{1}{5}} \alpha_{n} e_{n}(x)\right|^{2}\right)^{1 / 2}\right\|_{L^{5}\left([0, T]_{t} \times M_{x}\right)} \\
& \leq C\left\|\sum_{n}\left|e^{i t \lambda_{n}^{2}}\left(1+\lambda_{n}^{2}\right)^{\frac{s}{2}-\frac{1}{5}} \alpha_{n} e_{n}(x)\right|^{2}\right\|_{L^{5 / 2}\left([0, T]_{t} \times M_{x}\right)}^{1 / 2} \\
& \leq C\left(\sum_{n}\left\|\left|\left(1+\lambda_{n}^{2}\right)^{\frac{s}{2}-\frac{1}{5}} \alpha_{n} e_{n}(x)\right|^{2}\right\|_{L^{5 / 2}\left([0, T]_{t} \times M_{x}\right)}\right)^{1 / 2}
\end{aligned}
$$


Finally, using Proposition 6.1, we obtain

$$
\begin{aligned}
\left\|(-\boldsymbol{\Delta}+1)^{\frac{s}{2}-\frac{1}{5}} e^{i t \sqrt{-\boldsymbol{\Delta}}} f_{1}^{\omega}\right\|_{L^{5}\left(\Omega_{\omega} \times[0, T]_{t} \times M_{x}\right)} & \\
& \leq C T^{1 / 5}\left(\sum_{n}\left|\left(1+\lambda_{n}^{2}\right)^{s} \alpha_{n}\right|^{2}\right)^{1 / 2} \leq C T^{1 / 5}\left\|f_{1}\right\|_{H^{s}(M)} .
\end{aligned}
$$

The contribution of $f_{2}$ is dealt with similarly. This ends the proof of Proposition 6.4.

6.3. The fixed point. - In this section we shall prove only the case $s=8 / 21$ in Theorem 2. The case $s>8 / 21$ and the improved estimate for Gaussian are proved mutatis mutandi following the strategy developed in Section 5. Interpolating between (6.1) and the trivial bound

$$
\left\|u_{f}^{\omega}\right\|_{L^{2}\left(\Omega_{\omega} \times[0, T]_{t} \times M_{x}\right)} \leq C T^{1 / 2}\|f\|_{\mathcal{H}^{0}(M)}
$$

gives

$$
\left\|u_{f}^{\omega}\right\|_{L^{14 / 3}\left(\Omega_{\omega} \times[0, T]_{t} \times M_{x}\right)} \leq C T^{3 / 14}\|f\|_{\mathcal{H}^{8 / 21}(M)} .
$$

As in Section 5, we are looking for a fixed point of the map

$$
K_{f}^{\omega}: v \longmapsto-\int_{0}^{t} \frac{\sin ((t-\tau) \sqrt{-\boldsymbol{\Delta}})}{\sqrt{-\boldsymbol{\Delta}}}\left(\left(u_{f}+v\right)^{3}\right)(\tau, \cdot) d \tau .
$$

Using the Strichartz inequalities in Section 2 (with the new definition of admissible couples and consequently of $X_{T}^{s}$ and $Y_{T}^{s}$ spaces), we obtain

$$
\left\|K_{f}^{\omega}\right\|_{X_{T}^{2 / 3}} \leq C\left\|\left(u_{f}+v\right)^{3}\right\|_{Y_{T}^{1 / 3}} .
$$

But (observe that $(21 / 4,14 / 3)$ is a $2 / 3$-admissible couple)

$$
\|g\|_{L^{\infty}\left((0, T) ; H^{2 / 3}(M)\right)}+\|g\|_{L^{21 / 4}\left((0, T) ; L^{14 / 3}(M)\right)} \leq C\|g\|_{X_{T}^{2 / 3}}
$$

and (observe that $(21 / 2,14 / 5)$ is a $1 / 3$-admissible couple)

$$
\left\|v^{3}\right\|_{Y_{T}^{1 / 3}} \leq C\left\|v^{3}\right\|_{L^{21 / 19}\left((0, T) ; L^{14 / 9}(M)\right)} \leq C\|v\|_{L^{63 / 19}\left((0, T) ; L^{14 / 3}(M)\right)}^{3} \leq C_{T}\|v\|_{X_{T}^{2 / 3}}^{3} .
$$

These a priori bounds combined with the estimate (6.2) (and the fact that 21/4>63/19) allow to perform the fixed point in the space $X_{T}^{2 / 3}$ (for sufficiently small $T$ depending on $\omega)$, exactly as in the previous section. This ends the proof of Theorem 2 , 


\section{A}

\section{Ill posedness on $\mathcal{H}^{s}(M), s<1 / 2$.}

The goal of this appendix is to prove the ill-posedness statement claimed in the introduction. For that purpose, we first prove the lack of continuity at 0 of the flow map on $\mathcal{H}^{s}(M), s<1 / 2$. More precisely we have the following result.

Proposition A.1. - Let us fix $s \in] 0,1 / 2[$. Then there exists $\delta>0$ and a sequence $\left(t_{n}\right)_{n=1}^{\infty}$ of positive numbers tending to zero and a sequence $\left(u_{n}(t)\right)_{n=1}^{\infty}$ of $C^{\infty}(M)$ functions such that

$$
\left(\partial_{t}^{2}-\boldsymbol{\Delta}\right) u_{n}+u_{n}^{3}=0
$$

with

$$
\left\|u_{n}(0)\right\|_{\mathcal{H}^{s}(M)} \leq C \log (n)^{-\delta} \rightarrow_{n \rightarrow+\infty} 0
$$

but

$$
\left\|u_{n}\left(t_{n}\right)\right\|_{\mathcal{H}^{s}(M)} \geq C \log (n)^{\delta} \rightarrow_{n \rightarrow+\infty}+\infty .
$$

Proof. - The proof is strongly inspired by the considerations in [10] where the Euclidean space is considered instead of a riemannian manifold $(M, g)$. The only advantage of our argument with respect to [10] is that we avoid the scaling consideration of [10] and thus we can keep the argument local in space and thus it can still work for (1.1) posed on a compact manifold. A similar discussion in the context of NLS may be found in 4 .

Working in a local coordinate system near a fixed point of $M$, we consider an initial data concentrating at this fixed point. Namely, we consider (1.1) subject to initial conditions

$$
\left(f_{1, n}(x), f_{2, n}(x)\right)=\left(\kappa_{n} n^{\frac{3}{2}-s} \varphi(n x), 0\right), \quad n \gg 1,
$$

where $\varphi$ is a nontrivial bump function on $\mathbb{R}^{3}$ and

$$
\kappa_{n} \equiv[\log (n)]^{-\delta_{1}},
$$

with $\delta_{1}>0$ to be fixed later. The equation (1.1) being $H^{1}(M)$ sub critical (and defocusing), we obtain that (1.1) with data given by (A.1) has a unique global smooth solution which we denote by $u_{n}$. We will consider the solution of (1.1) with data (1.1) only for small times and thanks to the finite propagation speed of the wave equation the analysis is local. Next, let us denote by $V(t)$ the global solution of the ODE

$$
V^{\prime \prime}+V^{3}=0, \quad V(0)=1, V^{\prime}(0)=0 .
$$

Multiplying (A.2) by $V^{\prime}$, we deduce that $V(t)$ is a periodic function. We next denote by $v_{n}$ the solution of

$$
\partial_{t}^{2} v_{n}+v_{n}^{3}=0, \quad\left(v_{n}(0), \partial_{t} v_{n}(0)\right)=\left(\kappa_{n} n^{\frac{3}{2}-s} \varphi(n x), 0\right) .
$$


It is now clear that

$$
v_{n}(t, x)=\kappa_{n} n^{\frac{3}{2}-s} \varphi(n x) V\left(t \kappa_{n} n^{\frac{3}{2}-s} \varphi(n x)\right) .
$$

We next consider the semi-classical energy

$$
E_{n}(u) \equiv n^{-(1-s)}\left(\left\|\partial_{t} u\right\|_{L^{2}(M)}^{2}+\|\nabla u\|_{L^{2}(M)}^{2}\right)^{\frac{1}{2}}+n^{-(2-s)}\left(\left\|\partial_{t} u\right\|_{H^{1}(M)}^{2}+\|\nabla u\|_{H^{1}(M)}^{2}\right)^{\frac{1}{2}} .
$$

We are going to show that for very small times $u_{n}$ and $v_{n}$ are close with respect to $E_{n}$ but these small times are long enough to get the needed amplification of the $H^{s}$ norm (this amplification is a phenomenon only related to the solution of (A.3) ). Here is the precise statement.

Lemma A.2. - There exist $\varepsilon>0, \delta_{2}>0$ and $C>0$ such that, if we set

$$
t_{n} \equiv[\log (n)]^{\delta_{2}} n^{-\left(\frac{3}{2}-s\right)}
$$

then for every $n \gg 1$, every $t \in\left[0, t_{n}\right], E_{n}\left(u_{n}(t)-v_{n}(t)\right) \leq C n^{-\varepsilon}$. Moreover,

$$
\left\|u_{n}(t)-v_{n}(t)\right\|_{H^{s}(M)} \leq C n^{-\varepsilon} .
$$

Proof. - Set $w_{n}=u_{n}-v_{n}$. Then $w_{n}$ solves the equation

$$
\left(\partial_{t}^{2}-\boldsymbol{\Delta}\right) w_{n}=\boldsymbol{\Delta} v_{n}-3 v_{n}^{2} w_{n}-3 v_{n} w_{n}^{2}-w_{n}^{3}, \quad\left(w_{n}(0, \cdot), \partial_{t} w_{n}(0, \cdot)\right)=(0,0) .
$$

Set

$$
F \equiv \boldsymbol{\Delta} v_{n}-3 v_{n}^{2} w_{n}-3 v_{n} w_{n}^{2}-w_{n}^{3} .
$$

By the energy inequality inequality for the wave equation, we get

$$
\frac{d}{d t}\left(E_{n}\left(w_{n}(t)\right)\right) \leq C n^{-(2-s)}\|F(t, \cdot)\|_{H^{1}(M)}+C n^{-(1-s)}\|F(t, \cdot)\|_{L^{2}(M)} .
$$

We have for $t \in\left[0, t_{n}\right]$,

$$
\left\|\boldsymbol{\Delta}\left(v_{n}\right)(t, \cdot)\right\|_{H^{1}(M)} \leq C[\log (n)]^{3 \delta_{2}} n^{3-s}, \quad\left\|\boldsymbol{\Delta}\left(v_{n}\right)(t, \cdot)\right\|_{L^{2}(M)} \leq C[\log (n)]^{2 \delta_{2}} n^{2-s} .
$$

Therefore

$$
\frac{d}{d t}\left(E_{n}\left(w_{n}(t)\right)\right) \leq C\left([\log (n)]^{3 \delta_{2}} n+n^{-(2-s)}\|G(t, \cdot)\|_{H^{1}(M)}+n^{-(1-s)}\|G(t, \cdot)\|_{L^{2}(M)}\right)
$$

where

Writing for $t \in\left[0, t_{n}\right]$,

$$
G \equiv-3 v_{n}^{2} w_{n}-3 v_{n} w_{n}^{2}-w_{n}^{3}
$$

we obtain

$$
w_{n}(t, x)=\int_{0}^{t} \partial_{s} w_{n}(s, x) d s
$$

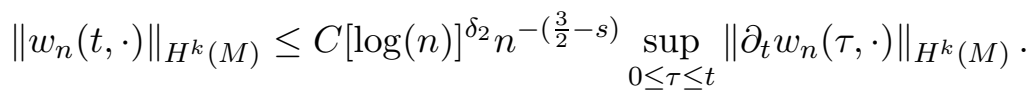


Moreover, we have that for $t \in\left[0, t_{n}\right]$,

$$
\left\|\nabla^{k} v_{n}(t, \cdot)\right\|_{L^{\infty}(M)} \leq C[\log (n)]^{k \delta_{2}} n^{\frac{3}{2}-s+k}
$$

and thanks to the Gagliardo-Nirenberg inequality,

$$
\left\|w_{n}(t, \cdot)\right\|_{L^{\infty}(M)} \leq C\left\|w_{n}(t, \cdot)\right\|_{H^{2}(M)}^{\frac{3}{4}}\left\|w_{n}(t, \cdot)\right\|_{L^{2}(M)}^{\frac{1}{4}} \leq C n^{\frac{3}{2}-s} E_{n}\left(w_{n}(t)\right) .
$$

Set

$$
e_{n}\left(w_{n}(t)\right) \equiv \sup _{0 \leq \tau \leq t} E_{n}\left(w_{n}(\tau)\right) .
$$

Using (A.6), (A.7) and (A.8), we get that for $l=1,2$,

$$
n^{-(l-s)}\|G(t, \cdot)\|_{H^{l-1}(M)} \leq C[\log (n)]^{l \delta_{2}} n^{\frac{3}{2}-s}\left(e_{n}\left(w_{n}\right)+\left[e_{n}\left(w_{n}\right)\right]^{3}\right) .
$$

Therefore, coming back to (A.5), we get

$$
\frac{d}{d t}\left(E_{n}\left(w_{n}(t)\right)\right) \leq C[\log (n)]^{3 \delta_{2}} n+C[\log (n)]^{l \delta_{2}} n^{\frac{3}{2}-s}\left(e_{n}\left(w_{n}\right)+\left[e_{n}\left(w_{n}\right)\right]^{3}\right) .
$$

We first suppose that $e_{n}\left(w_{n}(t)\right) \leq 1$ which holds for small values of $t$ since $w_{n}(0)=0$. Thanks to a Gronwall lemma argument for $t \in\left[0, t_{n}\right]$,

$$
e_{n}\left(w_{n}(t)\right) \leq C[\log (n)]^{\delta_{2}} n^{s-\frac{1}{2}} e^{C t[\log (n)]^{2 \delta_{2}} n^{\frac{3}{2}-s}} \leq C[\log (n)]^{\delta_{2}} n^{s-\frac{1}{2}} e^{C[\log (n)]^{2 \delta_{2}}} .
$$

(one should see $\delta_{2}$ as $3 \delta_{2}-2 \delta_{2}$ and $s-1 / 2$ as $1-(3 / 2-s)$ ). Since $s<1 / 2$, by taking $\delta_{2}>0$ small enough, we obtain that there exists $\varepsilon>0$ such that

$$
E_{n}\left(w_{n}(t)\right) \leq C n^{-\varepsilon}
$$

and in particular one has for $t \in\left[0, t_{n}\right]$,

$$
\left\|\partial_{t} w_{n}(t, \cdot)\right\|_{L^{2}(M)}+\left\|w_{n}(t, \cdot)\right\|_{H^{1}(M)} \leq C n^{1-s-\varepsilon} .
$$

We next estimate $\left\|w_{n}(t, \cdot)\right\|_{L^{2}}$. We may write for $t \in\left[0, t_{n}\right]$,

$$
\left\|w_{n}(t, \cdot)\right\|_{L^{2}(M)}=\left\|\int_{0}^{t} \partial_{t} w_{n}(s, \cdot)\right\|_{L^{2}(M)} \leq c t_{n} \sup _{0 \leq \tau \leq t}\left\|\partial_{t} w_{n}(\tau, \cdot)\right\|_{L^{2}(M)} .
$$

Thanks to (A.9) and the definition of $t_{n}$, we get

$$
\left\|w_{n}(t, \cdot)\right\|_{L^{2}(M)} \leq C[\log (n)]^{\delta_{2}} n^{-\left(\frac{3}{2}-s\right)} n^{1-s} n^{-\varepsilon} .
$$

Therefore, since $s<1 / 2$,

$$
\left\|w_{n}(t, \cdot)\right\|_{L^{2}(M)} \leq C n^{-s-\varepsilon} .
$$

An interpolation between (A.9) and (A.10) yields (A.4). This completes the proof of Lemma A.2. 
Using Lemma A.2, we may write

$$
\left\|u_{n}\left(t_{n}, \cdot\right)\right\|_{H^{s}(M)} \geq\left\|v_{n}\left(t_{n}, \cdot\right)\right\|_{H^{s}(M)}-C n^{-\varepsilon} .
$$

On the other hand from the representation of $v_{n}$, we obtain for $n$ large enough

$$
\left\|v_{n}\left(t_{n}, \cdot\right)\right\|_{H^{s}(M)} \geq C \kappa_{n}\left(t_{n} \kappa_{n} n^{\frac{3}{2}-s}\right)^{s}=C[\log (n)]^{-(s+1) \delta_{1}+s \delta_{2}} .
$$

Indeed this estimate is the consequence of the following lemma.

Lemma A.3. - Consider a smooth non constant $2 \pi$ periodic function $V$ and two functions $\psi, \phi \in C_{0}^{\infty}\left(\mathbb{R}^{d}\right)$ such that $\phi \psi$ is not identically vanishing. Then there exists $C>0$ such that for any $\lambda>1$ and any $s \geq 0$

$$
\|\psi(x) V(\lambda \phi(x))\|_{H^{s}\left(\mathbb{R}^{d}\right)} \geq \frac{\lambda^{s}}{C}-C .
$$

Proof. - The multiplication by a smooth function being continuous on $H^{s}$, it suffices to prove the estimate with $\psi$ replaced by any function $\chi \times \psi$ with $\chi \in C_{0}^{\infty}$. As a consequence (and using that $H^{s}$ is invariant by diffeomorphisms), we can assume that on the support of the function $\psi$, we have $\phi(x)=x_{1}$. We develop

$$
V(t)=\sum_{n \in \mathbb{Z}} v_{n} e^{i n t}, \quad\left|v_{n}\right| \leq C_{N}(1+|n|)^{-N}
$$

and replacing the function $V$ by $V-v_{0}$ (which changes the $H^{s}$ norm by at most a constant), we can assume $v_{0}=0$. Choose $n_{1} \neq 0$ such that $v_{n_{1}} \neq 0$ ( $V$ is non constant). Then

$$
\begin{aligned}
\left.\| \psi(x) V\left(\lambda x_{1}\right)\right) \|_{H^{s}\left(\mathbb{R}^{d}\right)}^{2}= & \int\left|\sum_{n} v_{n} \widehat{\psi}\left(\xi_{1}-n \lambda, \xi^{\prime}\right)\right|^{2}(1+|\xi|)^{2 s} d \xi \\
\geq & \int_{\left|\left(\xi_{1}-n_{1} \lambda, \xi^{\prime}\right)\right| \leq 1}\left|\sum_{n} v_{n} \widehat{\psi}\left(\xi_{1}-n \lambda, \xi^{\prime}\right)\right|^{2}(1+|\xi|)^{2 s} d \xi \\
\geq & \frac{1}{2} \int_{\left|\left(\xi_{1}-n_{1} \lambda, \xi^{\prime}\right)\right| \leq 1}\left|v_{n_{1}} \widehat{\psi}\left(\xi_{1}-n_{1} \lambda, \xi^{\prime}\right)\right|^{2}(1+|\xi|)^{2 s} \\
& \quad-2\left|\sum_{n \neq n_{1}} v_{n} \widehat{\psi}\left(\xi_{1}-n \lambda, \xi^{\prime}\right)\right|^{2}(1+|\xi|)^{2 s} d \xi
\end{aligned}
$$

The first term in the right hand side is bounded from below by

$$
\frac{1}{2} \int_{|\xi| \leq 1}\left|v_{n_{1}} \widehat{\psi}\left(\xi_{1}, \xi^{\prime}\right)\right|^{2}\left(1+\left|\xi_{1}+n_{1} \lambda\right|\right)^{2 s} \geq \frac{1}{C}\left|n_{1} \lambda\right|^{2 s}-C
$$


whereas the second term is bounded (in absolute value) by

$$
\begin{aligned}
2 \int_{|\xi| \leq 1}\left|\sum_{n \neq n_{1}} v_{n} \widehat{\psi}\left(\xi_{1}+\left(n_{1}-n\right) \lambda, \xi^{\prime}\right)\right|^{2}(1 & \left.+|\xi|+\left|n_{1} \lambda\right|\right)^{2 s} d \xi \\
& \leq C\left(\sum_{n \neq n_{1}}\left|v_{n}\right|\left|\left(n_{1}-n\right) \lambda-1\right|^{-N}\left|n_{1} \lambda\right|^{s}\right)^{2} \leq C
\end{aligned}
$$

which ends the proof of Lemma A.3.

By choosing $\delta_{1}$ small enough (depending on $\delta_{2}$ fixed in Lemma A.2), we obtain that $\lim _{n \rightarrow \infty}\left\|v_{n}\left(t_{n}, \cdot\right)\right\|_{H^{s}}=\infty$ which implies that $\lim _{n \rightarrow \infty}\left\|u_{n}\left(t_{n}, \cdot\right)\right\|_{H^{s}}=\infty$. This completes the proof of Proposition A.1.

We now can show that these solutions we just constructed can be glued together to give the following statement.

Proposition A.4. - Let us fix $s \in] 0,1 / 2\left[\right.$. There exists an initial data $f=\left(f_{1}, f_{2}\right) \in$ $\mathcal{H}^{s}(M)$ such there exists no solution of (1.1) in $L^{\infty}\left((-T, T) ; \mathcal{H}^{s}(M)\right), T>0$ with initial data $f$ satisfying in addition the finite speed of propagation.

Proof. - We use the notations introduced in the proof of the previous proposition and consider solutions $u_{n}$ as constructed above, but, working in local coordinates, with initial data centered at points $x_{n}=\left(x_{n, 1}, x_{n}^{\prime}=0\right)$ with a sequence $x_{n, 1}$ converging to 0 to be specified later. As a consequence, the support of the initial data of $u_{n}$ is included in the set

$$
\left\{x=\left(x_{1}, x^{\prime}\right) \in \mathbb{R}^{3}:\left|x_{1}-x_{n, 1}\right|+\left|x^{\prime}\right| \leq \frac{C}{n}\right\} .
$$

Furthermore, the explicit form of $v_{n}$ (and the fact that $t_{n} \ll n^{-1}$ ) shows that if

$$
K_{n}=\left\{x:\left|x_{1}-x_{n, 1}\right| \leq \frac{C}{2 n}-C t_{n}\right\}
$$

then

$$
\left\|u_{n}\left(t_{n}, \cdot\right)\right\|_{H^{s}\left(K_{n}\right)} \rightarrow+\infty
$$

Remark also that we have

Lemma A.5. - For any $0 \leq s<1 / 2$, there exists $C>0$ such that

$$
\forall n, \forall u \in H^{s}(M), \quad\|u\|_{H^{s}(M)} \geq C\left\|\left.u\right|_{K_{n}}\right\|_{H^{s}\left(K_{n}\right)} .
$$

Proof. - Indeed, the multiplication by the Heaviside function is continuous on $H^{s}(M)$ (because $-1 / 2<s<1 / 2$ and

$$
\left.u\right|_{K_{n}}=u \times 1_{-\frac{C}{2 n}+C t_{n}<x_{1}-x_{n, 1}<\frac{C}{2 n}-C t_{n}} .
$$


We now consider a sequence $\left(n_{k}\right)_{k \in \mathbb{N}}$ such that

$-\left\|u_{n_{k}}(0, \cdot)\right\|_{H^{s}(M)} \leq 2^{-k}$

$-n_{k} \leq 2^{-k}$.

Remark also that all the estimates on the functions $u_{n_{k}}$ are independent of the choice of the sequence $x_{n, 1}$ (because the bounds on $u_{n}$ we have are independent of the choice of the concentration point), and consequently, we can assume that $x_{n_{k}, 1}=\frac{1}{k^{2}}$. Consider now as initial data

$$
f=\left(f_{1}=\sum_{k \geq k_{0}} u_{n_{k}}(0), f_{2}=0\right),
$$

where $k_{0} \geq 1$ is a large constant. The support of the function $f_{1}$ is included in the union of the balls of radius $C 2^{-k}$ centered at $\left(\frac{1}{k^{2}}, 0,0\right)$. If $k_{0}$ is large enough, any solution $u$ of the non linear wave equation with initial data $f$, satisfying the finite speed of propagation will consequently coincide with the solutions $u_{n_{k}}, k \geq k_{0}$ we just constructed on the cone $K_{n_{k}}$ (notice that for $k_{0} \gg 1$, the cones $K_{n_{k}}, k \geq k_{0}$ are disjoint). As a consequence, these solutions will satisfy (using Lemma A.5)

$$
\left\|u\left(t_{n_{k}}, \cdot\right)\right\|_{\mathcal{H}^{s}(M)} \geq C\left\|u\left(t_{n_{k}}, \cdot\right)\right\|_{\mathcal{H}^{s}\left(K_{n_{k}}\right)} \rightarrow_{k \rightarrow+\infty}+\infty
$$

and consequently

$$
\limsup _{t \rightarrow 0^{+}}\|u(t, \cdot)\|_{\mathcal{H}^{s}(M)}=+\infty .
$$

This ends the proof of Proposition A.4

\section{B}

\section{Lack of $H^{s}$ regularization under the considered randomization}

The goal of this appendix is to give the proof of the following lemma.

Lemma B.1. - Let

$$
f=\sum_{n=1}^{\infty} \alpha_{n} e_{n}(x) \in H^{s}(M)
$$

be such that for some $\varepsilon>0$ one has that $f$ does not belong to $H^{s+\varepsilon}(M)$. Let $\left(l_{n}(\omega)\right)_{n=1}^{\infty}$ be a sequence of independent random variables such that there exists $c>0$ satisfying

$$
\limsup _{n \rightarrow+\infty} p\left(\left\{\left|l_{n}\right| \leq c\right\}\right)<1,
$$

(notice that this assumption is fulfilled if the random variables are identically distributed and non identically zero). If we set

$$
f^{\omega}=\sum_{n=1}^{\infty} l_{n}(\omega) \alpha_{n} e_{n}(x)
$$


then we have that $f^{\omega}$ belongs to $H^{s+\varepsilon}(M)$ with probability zero.

Proof. - A similar argument is given in [8]. Denote by $\mu_{n}$ the distribution of $\left(l_{n}(\omega)\right)_{n=1}^{\infty}$. By assumption there exists $c, \delta>0$ such that $\mu_{n}([-c, c]) \leq(1-\delta)$. Then, we can write (with $\rho_{n}=e^{-c^{2} \lambda_{n}^{2(s+\varepsilon)} \alpha_{n}^{2}}$ )

$$
\begin{gathered}
\int_{\Omega} e^{-\left\|f^{\omega}\right\|_{H^{s+\varepsilon}(M)}^{2}} d p(\omega)=\prod_{n=1}^{\infty} \int e^{-\lambda_{n}^{2(s+\varepsilon)} \alpha_{n}^{2} x^{2}} d \mu_{n}(x) \\
=\int_{-c}^{c} e^{-\lambda_{n}^{2(s+\varepsilon)} \alpha_{n}^{2} x^{2}} d \mu_{n}(x)+\int_{|x| \geq c} e^{-\lambda_{n}^{2(s+\varepsilon)} \alpha_{n}^{2} x^{2}} d \mu_{n}(x) \\
\leq \prod_{n=1}^{\infty}\left(\mu_{n}(-c, c)+\rho_{n}\left(1-\mu_{n}(-c, c)\right)\right)=\prod_{n=1}^{\infty}\left(\mu_{n}(-c, c)\left(1-\rho_{n}\right)+\rho_{n}\right) \\
\leq \prod_{n=1}^{\infty}\left((1-\delta)\left(1-\rho_{n}\right)+\rho_{n}\right) \leq \prod_{n=1}^{\infty}\left(1-\delta\left(1-\rho_{n}\right)\right) .
\end{gathered}
$$

Since by assumption $\sum_{n} \lambda_{n}^{2(s+\varepsilon)} \alpha_{n}^{2}=\infty$, we obtain that $\sum_{n=1}^{\infty}\left(1-e^{-c^{2} \lambda_{n}^{2(s+\varepsilon)}} \alpha_{n}^{2}\right)=\infty$ and therefore

$$
\prod_{n=1}^{\infty}\left(1-\delta\left(1-e^{-c^{2} \lambda_{n}^{2(s+\varepsilon)} \alpha_{n}^{2}}\right)=0 \Rightarrow \int_{\Omega} e^{-\left\|f^{\omega}\right\|_{H^{s+\varepsilon}(M)}^{2}} d p(\omega)=0 .\right.
$$

This implies that $\left\|f^{\omega}\right\|_{H^{s+\varepsilon}(M)}^{2}=\infty$ almost surely. This completes the proof of LemmaB.1.

\section{References}

[1] A. Ayache, N. Tzvetkov, $L^{p}$ properties of Gaussian random series, to appear in Trans. AMS.

[2] J. Bourgain, Periodic nonlinear Schrödinger equation and invariant measures, Comm. Math. Phys. 166 (1994) 1-26.

[3] J. Bourgain, Invariant measures for the 2D-defocusing nonlinear Schrödinger equation, Comm. Math. Phys. 176 (1996) 421-445.

[4] N. Burq, P. Gérard, N. Tzvetkov, Multilinear eigenfunctions estimates and global existence for the three dimensional nonlinear Schrödinger equations, Ann. ENS, 38 (2005) 255-301.

[5] N. Burq, P. Gérard and N. Tzvetkov. Two singular dynamics of the nonlinear Schrödinger equation on a plane domain, Geom. Funct. Anal., 13 (2003) 1 1-19.

[6] N. Burq, P. Gérard and N. Tzvetkov. An instability property of the nonlinear Schrödinger equation on $S^{d}$, Math. Res. Lett., 9:2-3 (2002) 323-335.

[7] N. Burq, G. Lebeau, F. Planchon, Global existence for energy critical waves in 3-d domains , Preprint, 2006. 
[8] N. Burq, N. Tzvetkov, Invariant measures for a three dimensional nonlinear wave equation, Preprint 2007 http://arxiv.org/abs/0707.1445.

[9] N. Burq, N. Tzvetkov, Random data Cauchy theory for supercritical wave equations II: A global result, Preprint 2007, http://arxiv.org/abs/070\%.1448.

[10] M. Christ, J. Colliander, T. Tao, Ill-posedness for nonlinear Schrödinger and wave equations, Preprint 2003.

[11] L. Kapitanskii, Some generalizations of the Strichartz-Brenner inequality, Leningrad Math. J. 1 (1990), 693-726.

[12] G. Lebeau, Perte de régularité pour les equations d'ondes sur-critiques, Bull. Soc. Math. France 133 (2005) 145-157.

[13] R.E.A.C. Paley, A. Zygmund, On some series of functions (1) (2) (3), Proc. Camb. Phil. Soc. 26 (1930) 337-357, 458-474, 28 (1932) 190-205.

[14] H. Smith, C. Sogge On the $L^{p}$ norm of spectral clusters for compact manifolds with boundary to appear in Acta Math. 2006.

[15] C. Sogge, Concerning the $L^{p}$ norm of spectral clusters for second order elliptic operators on compact manifolds, J. Funct. Anal. 77 (1988) 123-138.

[16] N. Tzvetkov, Invariant measures for the Nonlinear Schrödinger equation on the disc, Dynamics of PDE 3 (2006) 111-160.

[17] N. Tzvetkov, Invariant measures for the defocusing NLS, Preprint 2007.

[18] N. Tzvetkov, Construction of a Gibbs measure associated to the periodic Benjamin-Ono equation, Preprint 2006.

Nicolas Burq, Laboratoire de Mathématiques, Bât. 425, Université Paris Sud, 91405 Orsay Cedex, France et Institut Universitaire de France • E-mail : nicolas.burq@math.u-psud.fr

Nikolay Tzvetkov, Département de Mathématiques, Université Lille I, 59655 Villeneuve d'Ascq Cedex, France • E-mail : nikolay.tzvetkov@math.univ-lille1.fr 